\section{Elektrolytische Ätzung von Versetzungen auf Siliciumeisen mit Gosstextur}

Von W. D. Hannibal

Institut für Werkstoffe der Elektrotechnik der Technischen Hochschule Aachen

(Z. Naturforschg. 15 a, 837-838 [1960] ; eingegangen am 30. Mai 1960)

Die elektrolytische Ätzung von Versetzungen mit Chromsäure-Eisessig nach Morris ${ }^{1}$ auf Siliciumeisen ist in den letzten Jahren häufig angewandt worden ${ }^{2}$. Auch eine elektrolytische Ätzung mit einer 50-proz. wäßrigen Lösung von $\mathrm{CrO}_{3}$ sowie mit einem Chromsäure-Phosphorsäure-Elektrolyten $\left[88 \% \mathrm{H}_{3} \mathrm{PO}_{4} \quad\left(d=1,74 \mathrm{~g} / \mathrm{cm}^{3}\right)\right.$, $12 \% \mathrm{CrO}_{3}$ ] von $50{ }^{\circ} \mathrm{C}$ ist auf Siliciumeisen mit Erfolg benutzt worden ${ }^{3,4}$. Chromsäure-Phosphorsäure $\left[900 \mathrm{~cm}^{3}\right.$ $\left.\mathrm{H}_{3} \mathrm{PO}_{4}\left(d=1,71 \mathrm{~g} / \mathrm{cm}^{3}\right), 100 \mathrm{~g} \mathrm{CrO}{ }_{3}\right]$ von 20 bis $25{ }^{\circ} \mathrm{C}$ läßt sich unserer Erfahrung nach ebenfalls zur Sichtbarmachung von Versetzungen auf Siliciumeisen anwenden; dieser Elektrolyt hat aber den Nachteil, daß längere Ätzzeiten als bei Chromsäure-Eisessig erforderlich sind und zum Teil heterogene Einschlüsse ebenfalls herausgeätzt werden.

Úber eine direkte mikroskopische Beobachtung des Ätzvorgangs liegen meines Wissens keine Veröffentlichungen vor. Es soll daher im folgenden über Versetzungsätzungen mit zwei durchsichtigen Elektrolyten berichtet werden, die sich im elektrolytischen Poliergerät Elypovist der optischen Werke VEB Jena verwenden lassen. In diesem Poliergerät können Vorgänge auf der Metalloberfläche während des elektrolytischen Polierens und Ätzens mit 210 -facher Vergrößerung beobachtet werden ${ }^{5}$. Dieses Gerät ist grundsätzlich in folgender Weise aufgebaut: In einem Plastikbecher mit Zufluß und Abfluß ist am Boden eine Bohrung von $6 \mathrm{~mm} \varnothing$ angebracht, gegen die die Probe von unten gepreßt wird. Die Kathode in der Form eines konzentrischen Bechers aus nichtrostendem Stahl befindet sich wenige Millimeter über der Probe und hat ein Beobachtungsfenster aus Glas, so daß das Objektiv des Auflichtmikroskops vor Flüssigkeiten und Dämpfen geschützt ist. Die Proben werden im Naßschleifgerät von LunN ${ }^{6}$ vorgeschliffen.

Die beiden oben erwähnten chromsäurehaltigen Elektrolyte sind in diesem Mikroskop wegen Undurchsichtigkeit bzw. hoher Zähigkeit nicht anwendbar.

Die durchsichtigen Elektrolyte, mit denen wir Versetzungen auf $\mathrm{FeSi}$ unter dem Poliermikroskop anätzen konnten, haben folgende Zusammensetzung:

1 C. E. Morris, Metal Progress 56, 696 [1949].

2 Vgl. C. G. Dunn u. F. W. Daniels, Trans. Amer. Inst. Min. (Metall.) Engrs. 191, 147 [1951]. - C. G. Dunn u. W. R. Hibbard JR., Acta Met. 3, 409 [1955] ; 4, 306 [1956]. J. C. Surts u. J. R. Low JR., Acta Met. 5, 285 [1957]. C. G. Dunn u. E. F. Косн, Acta Met. 5, 548 [1957]. J. R. Low JR. u. R. W. Guard, Acta Met. 7, 171 [1959].

3 Vgl. B. Šesták, Czech. J. Phys. B 10, 91 [1960].

4 Vgl. B. Š Еsták, Czech. J. Phys. 8, 90 [1958].

5 Vgl. R. Zetzsche, Klepzig Fachberichte, Heft 7, S. 260 [1957]. - Vgl. Metal Treatment 26, 203 [1959].

6 Modell „Minor“ von H. Struers, Kopenhagen.
A) 33 Vol. $\% \mathrm{H}_{2} \mathrm{SO}_{4}, d=1,84 \mathrm{~g} / \mathrm{cm}^{3}$, 33 Vol. $\%$ Glyzerin, $34 \mathrm{Vol} . \% \mathrm{H}_{2} \mathrm{O}$ (s. Anm. ${ }^{7,8}$ ),
B) 48-proz. $\mathrm{H}_{3} \mathrm{PO}_{4}$ (s. Anm. ${ }^{9}$ ).

Beim Einschalten einer Spannung von rund 1 Volt fließt ein Strom von etwa $50 \mathrm{~mA}$ im Elektrolyten A, bzw. $80 \mathrm{~mA}$ in $\mathrm{B}$, entsprechend $175 \mathrm{~mA} / \mathrm{cm}^{2}$, bzw. $280 \mathrm{~mA} / \mathrm{cm}^{2}$. Es bildet sich zunächst eine braune Deckschicht, die sich nach kurzer Zeit ablöst und eine blanke Metalloberfläche sichtbar werden läßt, auf der unter einer zähflüssigen Deckschicht der Poliervorgang stattfindet. Bei etwa 1,2 Volt kommt es zum periodischen Wechsel von Passivierung und erneuter Aktivierung etc. Dieses Verhalten hängt von der Auslegung der in das Gerät eingebauten Stromversorgung ab. Kurz unterhalb dieser Spannung liegt das Optimum für einen Poliervorgang. Zur Ätzung auf der polierten Oberfläche ist nur die Spannung bis auf etwa 3 Volt zu erhöhen, wobei ein Strom von etwa $8 \mathrm{~mA}$, entsprechend rund $30 \mathrm{~mA} / \mathrm{cm}^{2}$, fließt und eine mäßige Sauerstoffentwicklung $\mathrm{zu}$ beobachten ist. Es werden nun Korngrenzen sichtbar und nach etwa 60 Sekunden die ersten Versetzungen bei Verwendung des Elektrolyten A, während es in B etwa 5 min dauert.

Die Ätzergebnisse mit A und B stimmen nahezu mit denen überein, die bei Ätzungen mit Chromsäure-Eisessig und kalter $\mathrm{H}_{3} \mathrm{PO}_{4}-\mathrm{CrO}_{3}$ in einer Apparatur mit rotierender Anode ${ }^{10}$ erhalten werden. Abb. ${ }^{*}$ zeigt

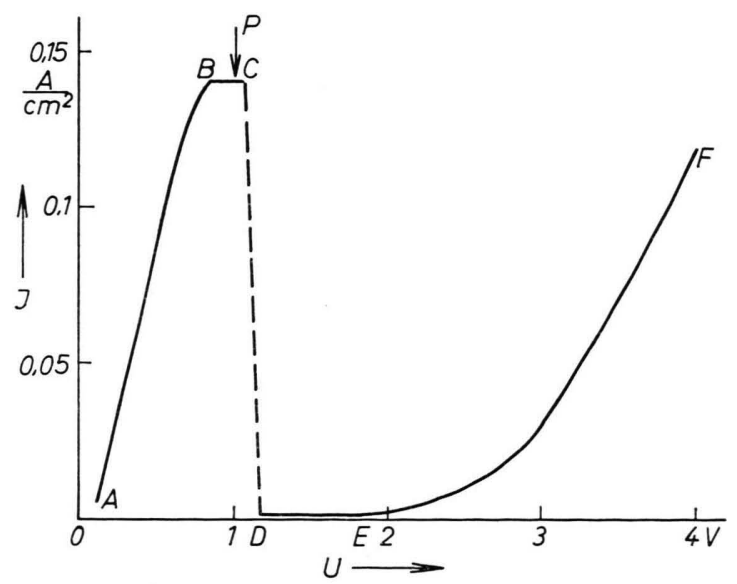

Abb. 2. Stationäre Stromdichte-Klemmspannungs-Kurve des Siliciumeisens im Poliermikroskop, Elektrolyt A.

7 Polierbad für Ni; vgl. W. MACHU u. A. RaGHeB, Z. Metallkunde 47, 176 [1956].

8 Elektrolyt A hat sich bei uns schon bewährt für die elektrolytische Politur von 50/50 Fe/Ni mit Würfeltextur und die Sichtbarmachung von Kleinwinkelkorngrenzen auf diesem Material (vgl. W. D. Hannibal, Acta Met. 7, 697 [1959]).

9 Polierbad für Fe; vgl. W. C. Elmore, J. Appl. Phys. 10, 724 [1939].

* Abb. 1 auf Tafel S. 836 a.

10 Vgl. C. E. Morris, l. c. ${ }^{1}$. - Vgl. L. F. Bates u. C. D. Mee, J. Sci. Instrum. 27, 317 [1950]. - Vgl. W. D. Hannibal, eine Vorveröffentlichung erscheint demnächst. 
schräg zur Metalloberfläche verlaufende Versetzungen in einer Kleinwinkelkorngrenze, die mit Elektrolyt B auf Siliciumeisen mit Gosstextur elektrolytisch angeätzt wurde. Die Korngrenze zwischen den einzelnen Kristalliten ist für eine Beobachtung im Auflicht-Phasenkontrast zu stark angeätzt.

Es stehen also zwei Gruppen von Elektrolyten zur Verfügung, mit denen man Versetzungen auf Siliciumeisen elektrolytisch ätzen kann:

1. $\mathrm{CrO}_{3}$-haltige Lösungen, wo durch $\mathrm{CrO}_{3}$ die Oberflächen passiert werden und beim Einschalten von 3 bis 5 Volt unter Sauerstoffentwicklung Stromdichten von rund $20 \mathrm{~mA} / \mathrm{cm}^{2}$ auftreten und

2. $\mathrm{CrO}_{3}$-freie Lösungen mit $\mathrm{SO}_{4}{ }^{--}$- oder $\mathrm{PO}_{4}{ }^{---}$-Ionen, bei denen anfänglich eine hohe Stromdichte $(>200 \mathrm{~mA} /$ $\mathrm{cm}^{2}$ ) nötig ist, um außerhalb der Spannungshöfe der Versetzungen den passiven Zustand zu erreichen, und dann eine geringere Stromdichte zur Passivitätserhaltung und Sauerstoffentwicklung $\left(20 \mathrm{~mA} / \mathrm{cm}^{2}\right)$.

Bei beiden Verfahren findet die Ätzung von Versetzungen im passiven Zustand bei gleichzeitiger Sauer-

11 Vgl. J. C. Surts u. J. R. Low JR., l. c. ${ }^{2}$.

12 Vgl. K. G. W $\mathrm{W}_{\mathrm{EIL}}$ u. D. Menzel, Z. Elektrochem. 63, 669 [1959].

13 Vgl. U. F. FranCK, Werkstoffe und Korrosion 9, 504 [1958]. stoffentwicklung statt. Es ist durchaus denkbar, daß der an den Versetzungen angereicherte Kohlenstoff, der bei FeSi zur Ätzbarkeit der Versetzungen nötig zu sein scheint ${ }^{11}$, die gleiche Rolle spielt wie $\mathrm{Cl}^{-}$-Ionen, die vermutlich die Deckschicht so stark stören, daß über Korngrenzen und Versetzungen die $\mathrm{Fe}^{++}$-Ionen durch sie hindurchwandern können ${ }^{12}$. Eine Aktivierung an diesen Stellen mit völliger Schichtzerstörung wird wohl nicht möglich sein, weil aktive Stellen auf der passiven Oberfläche nicht stabil sind ${ }^{12,13}$.

Bei Verwendung eines stabilisierten Netzgerätes ${ }^{\mathbf{1 4}}$ ließ sich die stationäre Strom-Klemmspannungs-Kurve im Poliermikroskop und in der Apparatur mit rotierender Anode ${ }^{11}$ aufnehmen. Sie verläuft beim Elektrolyten A (Abb. 2) ähnlich wie die stationäre StromdichteSpannungs-Kurve in potentiostatischer Schaltung von $\mathrm{Fe}$ in $1 n \cdot \mathrm{H}_{2} \mathrm{SO}_{4}{ }^{13,15}$.

Bei unseren Versuchen waren in der Nähe von $\mathrm{C}$ güntige Arbeitsbedingungen zum Polieren (P) während zwischen $\mathrm{E}$ und $\mathrm{F}$ eine kontrastreiche Ätzung von Versetzungen stattfand.

14 Das Netzgerät ist vom Typ AS 757 der Solartron Elektronik $\mathrm{GmbH}$ und liefert 0 bis 50 Volt stabilisierte Gleichspannung, Maximalstrom $1 \mathrm{~A}$.

15 Vgl. U. F. Franck, Habilitationsschrift, Göttingen 1954.

\section{Streuung von Galliumatomen in verschiedenen Zeeman-Zuständen}

\author{
Von K. Berkling, Ch. Schlier und P. Toschek
}

Physikalisches Institut der Universität Bonn

(Z. Naturforschg. 15 a, 838-839 [1960]; eingegangen am 4. August 1960)

Vor einigen Jahren wies RAMSAY ${ }^{1}$ darauf hin, daß der totale Streuquerschnitt von Atomen von ihrer gegenseitigen Orientierung abhängen sollte. Ein Atomstrahl, der durch eine Streukammer geschickt wird, sollte daher nicht nur abgeschwächt sondern auch teilweise ausgerichtet werden (collision alignment). Dabei ist es notwendig, daß die Strahlatome eine Drehimpulsquantenzahl $J \geqq 1$ haben.

Anstatt in einem durch Streuung geschwächten Atomstrahl nach der Ausrichtung zu suchen, kann man auch die Streuquerschnitte der Atome in den einzelnen ZEEMan-Zuständen bestimmen. Wir haben ein solches Experiment und zugehörige Rechnungen für die Kombination Gallium - Edelgas durchgeführt und die Differenz der totalen Querschnitte für die beiden Terme $m_{J}=+\frac{3}{2}$ und $m_{J}=+\frac{1}{2}$ des thermisch angeregten Zustandes ${ }^{2} \mathrm{P}_{3 / 2}$ gemessen. Diese Zustände werden im folgenden mit 33 und 31 bezeichnet.

Um systematische Fehler auszuschließen, bleibt die Geometrie der Anordnung von Spalten, Geschwindigkeitsselektor, Stern-Gerlach-Magnet, Streukammer und Langmuir-Taylor-Detektor während eines Versuchs ungeändert. Lediglich durch Umschalten des Magnetstroms werden die Zustände 33 und 31 abwechselnd durch das Spaltsystem durchgelassen. Der Detektorstrom wird in eine Impulsfolge verwandelt und diese in Zählern aufsummiert, die gleichsinnig mit dem Magneten umgeschaltet werden.

Um definierte Verhältnisse zu haben, ist die Streukammer in einem homogenen Magnetfeld angebracht, das quer zum Strahl gerichtet ist. Der 33- und 31-Strahl sind also transversal polarisiert.

Gemessen wurde das Intensitätsverhältnis $I_{31} / I_{33}$ für verschiedene Abschwächungen $I / I_{0}$ des Atomstrahls. Die Meßpunkte sind in Abb. 1 und 2 dargestellt. Für die Abschwächung $I / I_{0}=1 / e$ ist direkt $I_{31} / I_{33}=\left(\sigma_{31}-\sigma_{33}\right) / \sigma$. Die durch Mittelung erhaltenen Werte sind:

$$
\begin{array}{ll}
\mathrm{Ga}-\mathrm{Xe} & \left(\sigma_{31}-\sigma_{33}\right) / \sigma=(82 \pm 6) \cdot 10^{-4}, \\
\mathrm{Ga}-\mathrm{Ar} & \left(\sigma_{31}-\sigma_{33}\right) / \sigma=(65 \pm 5) \cdot 10^{-4}, \\
\mathrm{Ga}-\mathrm{He} & \left(\sigma_{31}-\sigma_{33}\right) / \sigma=(37 \pm 10) \cdot 10^{-4} .
\end{array}
$$

Diese Werte sind effektive Querschnitte, die mit den wirklichen Querschnitten für transversale Polarisation nur unter der Voraussetzung übereinstimmen, daß die Stoßrichtung jedes Stoßes gleich der Strahlrichtung ist. Dazu muß die Strahlgeschwindigkeit groß gegen die mittlere Geschwindigkeit der Streugasmoleküle sein, was für $\mathrm{Xe}$ gut, für $\mathrm{Ar}$ einigermaßen, für $\mathrm{He}$ jedoch gar nicht erfüllt ist. Der He-Wert sinkt daher auch, wie zu erwarten, ab.

Eine Berechnung des Effektes war bisher nur mit folgenden Approximationen möglich:

1. Nur das (winkelabhängige) van der WaALs-Potential zwischen den Atomen wird berücksichtigt.

1 N. F. Ramsay, Phys. Rev. 98, 1853 L [1955]. 